\title{
GENERALIZED COMBINATORIAL CELLS AND FACET SPLITTING
}

\author{
DAVID BARNETTE
}

\begin{abstract}
The similarity between triangulations of the sphere and simplicial polytopes makes cells with triangulated boundaries natural generalizations of simplicial polytopes. In this paper we extend this generalization to cells whose boundaries are broken up into more general structures than just simplices. These structures are called gcc's. In doing so we get a generalization of the $d$-polytope. We shall investigate a method of constructing these structures, called facet splitting. We show that almost all $d$-gcc's with up to $3+d$ facets can be constructed by facet splitting, and we construct a simple 4-gec with 10 facets that cannot be constructed in this way.
\end{abstract}

A convex polytope is the convex hull of a finite set of points. If it has dimension $d$, we shall call it a d-polytope. A $d$-polytope is simplicial provided all of its faces of dimension at most $d-1$ are simplices. Although the boundary of a simplicial $d$-polytope is a triangulation of the $(d-1)$-sphere, it is not true that every triangulation of the $(d-1)$-sphere is isomorphic to the boundary of a $d$-polytope.

2. Generalized combinatorial cells. We see that a $d$-polytope is a convex cell whose boundary consists of $k$-polytopes, $0 \leqq k \leqq d-1$, fitting together in a nice way. We use this idea to define generalized combinatorial cells.

A 0-generalized combinatorial cell (hereafter to be abbreviated gec) is a point. Inductively, a $d$-gcc $\mathscr{S}$ is a $d$-cell whose boundary is the union of a collection of $k$-gcc's called faces of $\mathscr{S},-1 \leqq k \leqq$ $d-1$ (a (-1)-gec is $\phi)$, satisfying the following:

(1) If $F$ is a face of $\mathscr{S}$ and $F_{1}$ is a face of $F$ then $F_{1}$ is a face of $\mathscr{S}$.

(2) If $F_{1}$ and $F_{2}$ are faces of $\mathscr{S}$ then $F_{1} \cap F_{2}$ is a face of $F_{1}$ and $F_{2}$ (note that $\phi$ is a face of $\mathscr{S}$ ).

A face $F_{1}$ of $\mathscr{S}$ is incident to a face $F_{2}$ provided either $F_{1} \subset F_{2}$ or $F_{2} \subset F_{1}$. Two gcc's $\mathscr{S}_{1}$ and $\mathscr{S}_{2}$ are isomorphic provided there is a one-to-one, dimension-preserving, incidence-preserving function from the set of faces of $\mathscr{S}_{1}$ onto the set of faces of $\mathscr{S}_{2}$. A facet of a $d$-gcc is a $(d-1)$-face, a subfacet is a $(d-2)$-face, a vertex is a 0 -face and an edge is a 1-face.

Clearly every 1 -gcc is isomorphic to a segment and every 2-gec 
is isomorphic to a polygon. It follows from a theorem of Steinitz [13] that every 3-gec is isomorphic to a 3-polytope. It is not true that every 4-gec is isomorphic to a 4-dimensional convex polytope (see [6] and [7]).

We shall now examine a method of constructing gec's. We shall begin by looking at 3-polytopes. Suppose we have a 3-polytope $P$ and we draw a segment across one of its facets. This segment divides the facet into two polygons, and its endpoints may also divide one or two edges into pairs of segments. It is easy to see that we have created a new 3-gec by adding this segment. We shall call this process facet splitting. A theorem of Steinitz [13] says that given any 3-polytope $P$, we may take a tetrahedron and obtain a 3 -gec isomorphic to $P$ by applying successive facet splittings. We shall extend the definition of facet splitting to all gec's.

Suppose $\mathscr{C}$ is a $d$-cell in $E^{n}$ and let $h$ be a homeomorphism of $\mathscr{C}$ onto the unit ball $B$ in $E^{d}$. We define $\operatorname{rel} \operatorname{int}(\mathscr{C})$ to be the inverse image of the interior of $B$ under $h$, and $\operatorname{rel} \beta(\mathscr{C})$ to be the inverse image of the boundary of $B$ under $h$.

Let $\mathscr{S}$ be a $d$-gec. We say that a $(d-1)$-cell $\mathscr{C}$ separates $\mathscr{S}$ provided $\mathscr{S} \sim \mathscr{C}$ has two components $X_{1}$ and $X_{2}$ such that $X_{1} \cup \mathscr{C}$ and $X_{2} \cup \mathscr{C}$ are $d$-cells.

We say that a $(d-1)$-cell $\mathscr{C}$ splits a $d$-gcc $\mathscr{S}$ provided

(3) $\operatorname{rel} \operatorname{Int}(\mathscr{C}) \subset \operatorname{rel} \operatorname{Int}(\mathscr{S})$ and $\operatorname{rel} \beta(\mathscr{C}) \subset \operatorname{rel} \beta(\mathscr{S})$,

(4) $\mathscr{C}$ separates $\mathscr{S}$,

(5) for every face $F$ of $\mathscr{S}, F \cap \mathscr{C}$ is a cell (possibly empty),

(6) if $\mathscr{C}$ meets a face $F$ of $\mathscr{S}$, then either $F \subset \mathscr{C}, F \cap \mathscr{C}$ is a face of $F$, or $\mathscr{C}$ separates $F$.

We shall now show that when $\mathscr{C}$ splits $\mathscr{S}$ it produces two $d$-gec's whose intersection is $\mathscr{C}$. To do this we first define the cells that will be the faces of the new gec's. Suppose $\mathscr{C}$ splits $\mathscr{S}$. We define inductively a collection of cells. Let $X_{1}$ and $X_{2}$ be the two components of $\mathscr{S} \sim \mathscr{C}$. If $e$ is an edge of $\mathscr{S}$ that is separated by $\mathscr{C}$ we define $e^{*}$ to be the 1-gec consisting of the segment $e \cap\left(X_{1} \cup \mathscr{C}\right)$ together with its endpoints. Inductively, if $\mathscr{C}$ separates a face $F$ of $\mathscr{S}$ we define $F^{*}$ to be the collection of all cells that are

(7) faces of $F$ that are subsets of $X_{1}$,

(8) cells of the form $F_{1} \cap \mathscr{C}$ where $F_{1}$ is a face of $F$, or

(9) cells of dimension less than the dimension of $F$ and are of the form $F_{2}^{*}$ for some face $F_{2}$ of $F$.

Starting with the other component of $\mathscr{S} \sim \mathscr{C}$ we can define a 1-gec $e^{* *}$ and then inductively faces $F^{* *}$ for any face $F$ of $\mathscr{S}$ that is separated by $\mathscr{C}$. We define $\mathscr{S}^{*}$ and $\mathscr{S}^{* *}$ the same way as we define $F^{*}$ and $F^{* *}$. 
Lemma 1. If a d-cell $\mathscr{C}$ splits a $(d+1)$-gcc $\mathscr{S}$, then $\mathscr{C}$ together with all cells of the form $\mathscr{C} \cap F$, where $F$ is a face of $\mathscr{S}$, forms a $d$-gec.

Proof. Our proof is by induction on $d$. The theorem is clearly true if $d=0$. For the inductive step we shall show first that $\operatorname{rel} \beta(\mathscr{C})$ is the union of $k$-gec's, $-1 \leqq k \leqq d-1$. Consider any cell of the form $\mathscr{C} \cap F$ where $F$ is a face of $\mathscr{S}$. If $\mathscr{C} \cap F=F$ or if $\mathscr{C} \cap F$ is a face of $F$ then $\mathscr{C} \cap F$ is a gec, thus we assume $\mathscr{C}$ separates $F$. In this case $\mathscr{C} \cap F$ splits $F$ and by induction $\mathscr{C} \cap F$ is a gec.

Suppose $x \in \operatorname{rel} \beta(\mathscr{C})$. By (3) $x \in \operatorname{rel} \beta(\mathscr{S})$ thus $x$ is in some face $F$ of $\mathscr{S}$ and thus is in some cell $\mathscr{C} \cap F$ in $\operatorname{rel} \beta(\mathscr{C})$. It follows that $\operatorname{rel} \beta(\mathscr{C})$ is the union of $k$-gcc's $-1 \leqq k \leqq d-1$. We shall call this collection of gec's $C$.

Next we wish to show that (1) and (2) hold for $C$. Suppose $F_{1}$ is a face of $\mathscr{C}$ and $F_{2}$ is a face of $F_{1}$. Then we can write $F_{1}=$ $G_{1} \cap \mathscr{C}$ where $G_{1}$ is a face of $\mathscr{S}$ and $F_{2}=G_{2} \cap\left(G_{1} \cap \mathscr{C}\right)$ where $G_{2}$ is a face of $G_{1}$. But now $F_{2}=G_{2} \cap \mathscr{C}$ which is a face of $\mathscr{C}$.

Suppose $F_{3}$ and $F_{4}$ are two faces of $\mathscr{C}$. We wish to show that $F_{3} \cap F_{4}$ is a face of $F_{3}$ and $F_{4}$. We may write $F_{3}=G_{3} \cap \mathscr{C}$ and $F_{4}=G_{4} \cap \mathscr{C}$ where $G_{3}$ and $G_{4}$ are faces of $\mathscr{S}$. Now $F_{3} \cap F_{4}=G_{3} \cap$ $\mathscr{C} \cap G_{4} \cap \mathscr{C}=G_{3} \cap G_{4} \cap \mathscr{C}$. Since $G_{3} \cap G_{4}$ is a face of $G_{3}$ and $G_{4}$ it follows that $F_{3} \cap F_{4}$ is a face of $F_{3}$ and $F_{4}$.

Lemma 2. $\mathscr{S}^{*}$ and $\mathscr{S}^{* *}$ are gcc's.

Proof. It suffices to deal with $\mathscr{S}^{*}$. Our proof will be by induction on $d$. It follows immediately by induction and Lemma 1 that all faces of $\mathscr{S}^{*}$ are gec's. It follows immediately from the definition of $S^{*}$ that if $F_{1}$ is a face of $F_{2}$ of $S^{*}$ then $F_{1}$ is a face of $S^{*}$. Let $F_{1}$ and $F_{2}$ be two faces of $S^{*}$ we wish to show that $F_{1} \cap F_{2}$ is a face of both. We treat several cases.

Case I. $\quad F_{1}$ and $F_{2}$ are faces of $\mathscr{S}$. The conclusion clearly follows in this case.

Case II. $F_{1}$ is a face of $\mathscr{S}, F_{2}$ is a face $G^{*}$ where $G$ is a face of $\mathscr{S}$. Since $F_{1}$ is a face of $\mathscr{S}$ we see that $G \cap F_{1}$ is not split by $\mathscr{C}$ thus $G \cap F_{1}$ is a face of $\mathscr{S}^{*}$ and the conclusion follows.

Case III. $F_{1}$ is a face of $\mathscr{S}, F_{2}$ is a face of $\mathscr{C}$. Since $\mathscr{C}$ does not split $F_{1}$ we see that $F_{1} \cap \mathscr{C}$ is a face of $F_{1}$ and also a face of $\mathscr{C}$, and thus is a face of $F_{2}$. 
Case IV. $F_{1}$ is a face $G^{*}$, where $G$ is a face of $\mathscr{S}$, and $F_{2}$ is a face of $\mathscr{C}$. In this case $F_{1} \cap F_{2}=G^{*} \cap G_{2} \cap \mathscr{C}$ where $G_{2}$ is a face of $\mathscr{S}$. But $G^{*} \cap \mathscr{C}=G \cap \mathscr{C}$ thus $F_{1} \cap F_{2}=G \cap G_{2} \cap \mathscr{C}$ which is a face of $\mathscr{S}^{*}$.

Case V. $F_{1}=G_{1}^{*}$ and $F_{2}=G_{2}^{*}$ where $G_{1}$ and $G_{2}$ are faces of $\mathscr{S}$. The same type of argument as in Case IV suffices.

Case VI. $F_{1}$ and $F_{2}$ are faces of $\mathscr{C}$. The conclusion follows from Lemma 1.

It is not true that if we split a facet of a $d$-gec that we will always produce another $d$-gcc. To see this, suppose that we have a 4-gcc $P$ with four facets meeting at an edge $e$. Let $\mathscr{F}_{1}$ be one of these facets and let $\mathscr{F}_{2}$ be the facet whose intersection with $\mathscr{F}_{1}$ is $e$. If we split $\mathscr{F}_{1}$ in such a way that we split $e$ then the result in $\mathscr{F}_{2}$ is that we split $e$ and no other face. As a result of the splitting, $\mathscr{F}_{2}$ becomes something other than a 3-gec thus the splitting does not produce a 4 -gec.

We shall need the following definition and lemmas in order to describe splittings that do produce gec's.

We say that a $d$-gce $\mathscr{S}$ is simple at a $k$-face $F$ provided $F$ lies in exactly $d-k$ facets of $\mathscr{S}$.

Lemma 3. Let $F$ be a face of $a d$-gec $\mathscr{S}$ and let $F_{1}$ be a facet of $F$. Then there exists a facet $\mathscr{F}$ of $\mathscr{S}$ such that $F_{1} \subset \mathscr{F}$ and $F \not \subset \mathscr{F}$.

Proof. Our proof is by induction on $d$. Let $\mathscr{F}_{1}$ be a facet of $\mathscr{S}$ containing $F$.

Case I. $F=\mathscr{F}_{1}$. Let $x \in$ rel int $F_{1}$ and let $N$ be a neighborhood of $x$ in $\operatorname{rel} \beta(\mathscr{S})$. Since $x \in \operatorname{rel} \beta(F)$ we see that there are points in $N$ that do not belong to $F$ thus some facet meets $F$ on $F_{1}$. This is the desired facet.

Case II. $F \neq \mathscr{F}_{1}$. By induction there is a facet $F_{2}$ of $\mathscr{F}_{1}$ containing $F_{1}$ but not containing $F$. Let $\mathscr{F}_{2}$ be the facet of $\mathscr{S}$ meeting $\mathscr{F}_{1}$ on $F_{2}$. The facet $\mathscr{F}_{2}$ is the desired facet.

Lemma 4. If $F$ is a $k$-face of $a d$-gec $\mathscr{S}$ then $F$ belongs to at least $d-k$ facets of $\mathscr{S}$.

Proof. Our proof is by induction on $d$. Let $\mathscr{F}$ be a facet of $\mathscr{S}$ containing $F$. By induction $F$ lies in at least $d-k-1$ facets 
of $\mathscr{F}$. Each of these facets is the intersection of $\mathscr{F}$ with another facet of $\mathscr{S}$. Thus $F$ belongs to at least $d-k$ facets of $\mathscr{S}$.

Lemma 5. If a $d$-gec is simple at a $k$-face $F$ then it is simple at any $j$-face $F_{1}$ containing $F$.

Proof. Suppose $F_{1}$ lies in more than $d-j$ facets. Let $G_{0}, G_{1}$, $G_{2}, \cdots, G_{n}$ be a sequence of faces of $\mathscr{S}$ such that each face is a facet of its predecessor, each contains $F, G_{0}=F_{1}$ and $G_{n}=F$. Then each $G_{i}$ lies in at least $d-j+i+1$ facets. Since $n=j-k$ we have that $F$ lies in at least $d-j+(j-k)+1=d-k+1$ facets which is a contradiction.

COROLlaRY. If $\mathscr{S}$ is simple at each vertex then it is simple at every face.

If $\mathscr{S}$ is simple at each vertex we say that $\mathscr{S}$ is a simple gec.

Lemma 6. If a $d$-gec $\mathscr{S}$ is simple at a $j$-face $F$ then the intersection of any $k$ facets of $\mathscr{S}$ containing $F$ is exactly $d-k$ dimensional.

Proof. Let $F_{1}$ be the intersection of facets $\mathscr{F}_{1}, \cdots, \mathscr{F}_{k}$ with $F$ a face of each $\mathscr{F}_{2}$. Since $\mathscr{S}$ is simple at $F_{1}, F_{1}$ lies in exactly $d-l$ facets, where $l$ is the dimension of $F_{1}$. Thus $d-l \geqq k$ or $l \leqq d-k$.

Suppose $l=d-k-r$ for some positive integer $r$. Let $F_{2}$ be a facet of $F_{1}$ containing $F$ and let $\mathscr{F}_{1}^{\prime}$ be a facet of $\mathscr{S}$ containing $F_{2}$ but not $F_{1}$. Let $F_{3}$ be a facet of $F_{2}$ containing $F$ and let $\mathscr{F}_{2}^{\prime}$ be a facet of $\mathscr{S}$ containing $F_{3}$ but not $F_{2}$. We continue in this way until we have a sequence of facets $\mathscr{F}_{1}^{\prime}, \mathscr{F}_{2}^{\prime}, \cdots, \mathscr{F}_{n}{ }^{\prime}$ and a sequence of faces $F_{1}, \cdots, F_{n-1}=F$.

The face $F_{n-1}$ has dimension $d-k-n=j$ thus if we intersect $k+n=d-j-r$ facets of $\mathscr{S}$ we get a face containing $F$. But this is impossible because $F$ lies in exactly $d-j$ faces.

Lemma 7. If a $d$-gec $\mathscr{S}$ is simple at a face $F$, if $\mathscr{F}$ is a facet of $\mathscr{S}$ containing $F$ and if $F_{1}$ is a face containing $F$ and not lying in $\mathscr{F}$ then $\mathscr{F} \cap F_{1}$ is a facet of $F_{1}$.

Proof. Let the dimension of $F$ be $k$. By Lemma 5, $\mathscr{S}$ is simple at $F_{1}$. By Lemmas 4 and $6, F_{1}$ is the intersection of $d-k$ facets of $\mathscr{S}$, thus $\mathscr{F} \cap F_{1}$ is the intersection of $d-k+1$ facets. By Lemma $6, \mathscr{F} \cap F_{1}$ is a $(k-1)$-face of $F_{1}$. 
LEMMA 8. If $\mathscr{C}$ splits a gec $\mathscr{S}$ and splits a face $F$ of $\mathscr{S}$ then $\mathscr{C}$ splits every face containing $F$.

Proof. Let $F_{1}$ be a face containing $F$. Clearly $F_{1} \not \subset \mathscr{C}$ because $F \not \subset \mathscr{C}$. Also $F_{1} \cap \mathscr{C}$ is not a face of $F_{1}$ because then $F_{1} \cap \mathscr{C}$ would be either a face of $F$ or a face containing $F$ which would imply that $\mathscr{C}$ does not split $F$. Thus by (6), $\mathscr{C}$ splits $F_{1}$.

LEMMA 9. If $\mathscr{S}$ is simple at $F, \mathscr{C}$ splits a facet $\mathscr{F}$ containing $F, \mathscr{C}$ splits $F$, and $F_{1}$ is a face of $\mathscr{S}$ containing $F$ and not lying in $\mathscr{F}$, then $\mathscr{C}$ splits a facet of $F_{1}$.

Proof. By Lemma $7, F_{1} \cap \mathscr{F}$ is a facet of $F_{1}$. But $\mathscr{C}$ splits $\mathscr{F}$ and $F$ thus by Lemma $8, \mathscr{C}$ splits $F_{1} \cap \mathscr{F}$.

With these lemmas out of the way we can now talk about splittings which produce gec's.

If a cell $\mathscr{C}$ splits a facet $\mathscr{F}$ of a $d$-gcc $\mathscr{S}$ in such a way that whenever $\mathscr{C}$ splits a face $F$ of $\mathscr{S}$ then $\mathscr{S}$ is simple at $F$, then we are able to define a new gec. The faces of the new gec are of the following types:

(10) Faces of the form $F^{*}$ and $F^{* *}$ and their faces, for every face $F$ of $\mathscr{S}$ split by $\mathscr{C}$.

(11) Faces of $\mathscr{S}$ that are not of the form $F^{*}$ or $F^{* *}$ but have faces split by $\mathscr{C}$.

(12) Faces of $\mathscr{S}$ that do not have faces split by $\mathscr{C}$.

We shall call this collection of gcc's $\mathscr{S}+\mathscr{C}$. We shall say that $\mathscr{C}$ splits a facet of $\mathscr{S}$ properly if it splits as described above.

THEOREM 1. If $\mathscr{C}$ splits a facet $\mathscr{F}$ of a $d$-gec $\mathscr{S}$ properly then $\mathscr{S}+\mathscr{C}$ is a d-gcc.

Proof. Our proof is by induction on $d$. It is easily seen that the theorem is true for $d \leqq 2$.

By Lemma 2, faces of type (10) are gcc's. Suppose $F$ is a face of $\mathscr{S}$ that has a face that is split by $\mathscr{C}$ and that $F$ is not split by $\mathscr{C}$. By Lemma 8, $F$ does not lie in $\mathscr{F}$. By Lemma $9, \mathscr{C}$ splits a facet of $F$. By induction, splitting a facet of $F$ produces a gcc. Thus faces of type (11) are gcc's. Clearly faces of type (12) are gcc's.

Checking that (1) and (2) hold for $\mathscr{S}+\mathscr{C}$ is routine and is left to the reader.

If $\mathscr{S}^{\prime}=\mathscr{S}+\mathscr{C}$ then we also write $\mathscr{S}=\mathscr{S}^{\prime}-\mathscr{C}$. If $\mathscr{C}$ is a subfacet of $\mathscr{S}$ such that $\mathscr{S}-\mathscr{C}$ is a gec we say that $\mathscr{C}$ is a removable subfacet of $\mathscr{S}$. 
3. Generating combinatorial cells. As we have mentioned, the combinatorial types of 3-gcc's can be generated from the tetrahedron by facet splitting. In [8] we show that the combinatorial types of simple 4-polytopes with up to 8 facets can be generated from the 4-simplex by facet splitting. We conjecture that all $d$-gcc's with up to $d+3$ facets can be generated from the $d$-simplex by facet splitting. Proving this is surprisingly difficult. In fact, our results in this paper fall slightly short of this conjecture.

In order to prove our main theorem on generating gec's we need the following definitions and lemmas

If $v$ is a vertex of a $d$-gec $\mathscr{S}$ then we define $\operatorname{star}(v, \mathscr{S})$ to be the collection of faces of $\mathscr{S}$ meeting $v$ and faces of faces meeting $v$. We define ast $(v, \mathscr{S})$ to be the collection of all faces of $\mathscr{S}$ that miss $v$. We define $\operatorname{link}(v, \mathscr{S})$ to be ast $(v, \mathscr{S}) \cap \operatorname{star}(v, \mathscr{S})$. We shall also use ast $(v, \mathscr{S}), \operatorname{star}(v, \mathscr{S})$, and $\operatorname{link}(v, \mathscr{S})$ to denote the union of faces in ast $(v, \mathscr{S})$, $\operatorname{star}(v, \mathscr{S})$, and link $(v, \mathscr{S})$ respectively. It will be clear from the context which meaning we are using.

LEMMA 10. If $v$ is a vertex of a simple $d$-gcc $\mathscr{S}$ with at most $d+3$ facets then link $(v, \mathscr{S})$ is a $(d-2)$-sphere and ast $(v, \mathscr{S})$ is a $(d-1)$-cell.

Proof. Our proof is by induction on $d$. The theorem is clearly true if $d \leqq 2$. We thus assume that the theorem is true for all $k$ gec's $k<d$.

Consider the collection $A$ of all sets of the form ast $(v, H)$ where $H$ is a face of $\mathscr{S}$ meeting $v$. By induction these sets are cells. We shall show that this collection is isomorphic to the boundary of the $(d-1)$-simplex by giving an explicit isomorphism. Let $T$ be the $(d-1)$-simplex with facets $R_{1}, \cdots, R_{d}$. Let $\mathscr{F}_{1}, \cdots, \mathscr{F}_{d}$ be the facets of $\mathscr{S}$ meeting $v$. We map ast $\left(v, \mathscr{F}_{i}\right)$ onto $R_{i}$.

If $F$ is a $k$-face of $\mathscr{S}$ meeting $v$ then $F$ is the intersection of exactly $d-k$ facets of $\mathscr{S}$. We map ast $(v, F)$ onto the intersection of the corresponding $d-k$ facets in $T$. It is easy to check that this is an isomorphism, thus the union of the cells of $A$ is a sphere, but this union is just link $(v, S)$.

Let $\mathscr{F}_{1}^{\prime}, \mathscr{F}_{2}^{\prime}$, and $\mathscr{F}_{3}^{\prime}$ be the facets of $\mathscr{S}$ in ast $(v, \mathscr{S})$. If two of these facets do not meet or if all three intersect on a $(d-3)$-face then it is easy to see that ast $(v, \mathscr{S})$ is a cell and that link $(v, \mathscr{S})$ is a sphere.

Suppose on the other hand that the three subfacets of $\mathscr{S}, \mathscr{F}_{1}^{\prime} \cap$ $\mathscr{F}_{2}^{\prime}, \mathscr{F}_{2}^{\prime} \cap \mathscr{F}_{3}^{\prime}$, and $\mathscr{F}_{3}^{\prime} \cap \mathscr{F}_{1}^{\prime}$ are pairwise disjoint. Then the boundary of $\mathscr{F}_{1}^{\prime} \cup \mathscr{F}_{2}^{\prime} \cup \mathscr{F}_{3}^{\prime}$ is homeomorphic to a cylinder over a $(d-2)$ sphere, with the bases of the cylinder identified. There are two 
identification homeomorphisms, one orientation preserving and one orientation reversing. Thus $\beta\left(\mathscr{F}_{1} \cup \mathscr{F}_{2} \cup \mathscr{F}_{3}\right)$ is either nonorientable or homeomorphic to the product of a $(d-2)$-sphere with a 1-sphere, in either case it is not a sphere which is a contradiction. It should be noted that this argument works only for $d \geqq 4$, for in the case $d=3, \mathscr{F}_{1} \cup \mathscr{F}_{2} \cup \mathscr{F}_{3}$ could be a mobius strip, whose boundary is a 1-sphere. But in this case it is well known that link $(v, \mathscr{S})$ is a 1 -sphere and ast $(v, \mathscr{S})$ is a 2-cell.

If $v$ is a vertex of a simple $d$-gec $\mathscr{S}$, with at most $d+3$ facets we can construct a related $d$-gcc which we denote by $\mathscr{S}^{\sharp}$.

If $d \leqq 1$ then $\mathscr{S}^{\sharp}=\mathscr{S}$. Inductively, we define the faces of $\mathscr{S}^{\sharp}$ as follows:

(13) Faces of the form $\mathscr{F}^{*}$ where $\mathscr{F}$ meets $v$.

(14) Faces of faces of type (13).

(15) The antistar of $v$ in $\mathscr{S}$.

THEOREM 2. $\mathscr{S}^{\ddagger} i$ s a $d$-gec isomorphic to the $d$-simplex.

The proof is a routine inductive proof and will be eliminated here.

We shall need the gec equivalent of prisms and pyramids. Let $\mathscr{S}_{1}$ and $\mathscr{S}_{2}$ be two copies of a $d$-gec $\mathscr{S}$ in parallel hyperplanes in $E^{d+1}$ with $\mathscr{S}_{2}$ a translate of $\mathscr{S}_{1}$. For each face $F$ of $\mathscr{S}$ let $F^{\prime}$ be the union of all segments joining points in the faces in $\mathscr{S}_{1}$ and $\mathscr{S}_{2}$ corresponding to $F$. Let $\mathscr{S}^{\prime}$ be the union of all segments joining points in $\mathscr{S}_{1}$ with points in $\mathscr{S}_{2}$. It is easily verified that $\mathscr{S}^{\prime}$ together with all cells of the form $F^{\prime}$ form a $(d+1)$-gce. This $(d+1)$-gce and any $(d+1)$-gcc isomorphic to it are called the prism over $\mathscr{S}$, or the prism with base $\mathscr{S}$. The copies of $\mathscr{S}$ are called the bases of the prism.

Let $\mathscr{S}$ be a $d$-gec in $E^{d}$ and let $x$ be a point in $E^{d+1} \sim E^{d}$. For each face $F$ of $\mathscr{S}$ we define $F^{\prime \prime}$ to be the union of all segments from $x$ to points in $F$. We define $\mathscr{S}^{\prime \prime}$ to be the union of all segments from $x$ to points in $\mathscr{S}$. It is easily verified that $\mathscr{S}^{\prime \prime}$ together with all cells of the form $F^{\prime \prime}$ forms a $(d+1)$-gec. This $(d+1)$-gcc and any $(d+1)$-gcc isomorphic to it are called the pyramid over $\mathscr{S}$, or the pyramid with apex $x$ and base $\mathscr{S}$. If we take a $d$-gec $\mathscr{S}$, take a pyramid over it, take a pyramid over this $(d+1)$-gec and continue this process $k$ times we get a $(d+k)$-gec called the $k$-fold pyramid over $\mathscr{S}$.

Lemma 11. If $\mathscr{S}$ is a simple d-gce with two disjoint facets $\mathscr{F}_{1}$ and $\mathscr{F}_{2}$ such that every vertex of $\mathscr{S}$ lies on $\mathscr{F}_{1}$ or $\mathscr{F}_{2}$ then $\mathscr{S}$ is a prism with bases $\mathscr{F}_{1}$ and $\mathscr{F}_{2}$. 
Proof. Our proof is by induction on $d$. Let $\mathscr{F}$ be a facet of $\mathscr{S}$ other than $\mathscr{F}_{1}$ or $\mathscr{F}_{2}$. Since $\mathscr{S}$ is simple, $\mathscr{F} \cap \mathscr{F}_{1}$ is either empty or a facet of $\mathscr{F}$. If $\mathscr{F} \cap \mathscr{F}_{1}$ were empty this would imply that all vertices of $\mathscr{F}$ are vertices of $\mathscr{F}_{2}$. Thus $\mathscr{F} \cap \mathscr{F}_{1}$ is a facet of $\mathscr{F}$ and so is $\mathscr{F} \cap \mathscr{F}_{2}$. By induction $\mathscr{F}$ is a prism with bases $\mathscr{F} \cap \mathscr{F}_{1}$ and $\mathscr{F} \cap \mathscr{F}_{2}$. It follows that every face of $\mathscr{S}$ not on $\mathscr{F}_{1}$ or $\mathscr{F}_{2}$ is a prism with one base in $\mathscr{F}_{1}$ and one base in $\mathscr{F}_{2}$. This provides an obvious isomorphism of $\mathscr{S}$ with the prism over $\mathscr{F}_{1}$.

We define a simple $d$-gce to be 2-neighborly provided each two facets meet (and therefore meet on a subfacet). We say that a triangulation of the $d$-sphere is 2-neighborly provided each two vertices are joined by an edge. There is no ambiguity since the only simple triangulation of the $d$-sphere is the $(d+1)$-simplex.

We are now ready for our main theorems on generating gec's.

Theorem 3. If $\mathscr{S}$ is a simple $d$-gec with $d+2$ facets or if $\mathscr{S}$ is a non 2-neighborly simple $d$-gec with $d+3$ facets then $\mathscr{S}=\mathscr{S}^{\prime}+$ $\mathscr{C}$ for some subfacet $\mathscr{C}$ of $\mathscr{S}$ and some d-gec $\mathscr{S}^{\prime}$.

Proof. We shall give the proof for the case where $\mathscr{S}$ is non 2-neighborly and has $d+3$ facets. The proof in the other case is similar. Let $\mathscr{F}_{1}$ and $\mathscr{F}_{2}$ be two facets of $\mathscr{S}$ that do not meet. We consider two cases.

Case I. All vertices of $\mathscr{S}$ lie on either $\mathscr{F}_{1}$ or $\mathscr{F}_{2}$. In this case $\mathscr{S}$ is a prism over the $(d-1)$-gcc $\mathscr{F}_{1}$ which by induction has a subfacet $\mathscr{C}^{\prime}$ such that $\mathscr{F}_{1}=\mathscr{F}_{1}^{\prime}+\mathscr{C}^{\prime}$ for some $(d-1)$-gec $\mathscr{F}_{1}^{\prime}$. Now if $\mathscr{C}$ is a prism over $\mathscr{C}^{\prime}$ and if $\mathscr{S}^{\prime}$ is a prism over $\mathscr{F}_{1}^{\prime}$ then $\mathscr{S}=\mathscr{S}^{\prime}+\mathscr{C}$.

Case II. Some vertex $v$ of $\mathscr{S}$ misses $\mathscr{F}_{1}$ and $\mathscr{F}_{2}$. In this case $\mathscr{F}_{1}$ and $\mathscr{F}_{2}$ are in ast $(v, \mathscr{S})$ and by Lemma 10 we have that there is a facet $\mathscr{F}_{3}$ in ast $(v, \mathscr{S})$ such that $\mathscr{F}_{1} \cap \mathscr{F}_{3}$ is a subfacet, $\mathscr{F}_{2} \cap$ $\mathscr{F}_{3}$ is a subfacet and $\left(\mathscr{F}_{1} \cap \mathscr{F}_{3}\right) \cap\left(\mathscr{F}_{2} \cap \mathscr{F}_{3}\right)=\varnothing$.

We wish to show that $\mathscr{F}_{1} \cap \mathscr{F}_{3}$ splits a facet of $\mathscr{S}^{\sharp}$, namely the facet ast $(v, \mathscr{S})$. The only conditions on $\mathscr{F}_{1} \cap \mathscr{F}_{3}$ that are not clearly true are (5) and (6).

Consider any set of the form $\mathscr{F}_{1} \cap \mathscr{F}_{3} \cap F$ where $F$ is a face of $\mathscr{S}^{\sharp}$ on ast $(v, \mathscr{S})$. Any such set is also the set $\mathscr{F}_{1} \cap \mathscr{F}_{3} \cap F^{\prime \prime}$ where $F^{\prime}$ is the face of $\mathscr{S}^{\sharp}$ of smallest dimension that contains $v$ and $F$. But since $\mathscr{F}_{1} \cap \mathscr{F}_{3}$ intersected with any face of $\mathscr{S}$ containing $v$ is a cell, it follows that $\mathscr{F}_{1} \cap \mathscr{F}_{3} \cap F$ is a cell.

Suppose $\mathscr{F}_{1} \cap \mathscr{F}_{3}$ meets a face $F_{1}$ of $\mathscr{S}$. Since $\mathscr{S}$ is simple we see that $F_{1} \not \subset \mathscr{F}_{1} \cap \mathscr{F}_{3}$. For the same reason $F_{1} \cap \mathscr{F}_{1} \cap \mathscr{F}_{3}$ is not a 
face of $F_{1}$ thus we must show that $\mathscr{F}_{1} \cap \mathscr{F}_{3}$ separates $F_{1}$. The face $F_{1}$ is a union of faces of $\mathscr{S}$, say $F_{2}, F_{3}, \cdots, F_{n}$. There can be at most three such faces, one belonging to $\mathscr{F}_{1}$, one belonging to $\mathscr{F}_{2}$ and one belonging to $\mathscr{F}_{3}$. The set $F_{1} \sim\left(\mathscr{F}_{1} \cap \mathscr{F}_{3}\right)$ will contain one of these faces in one component and two, say $F_{2}$ and $F_{3}$, in the other. Thus we need to show that $F_{2} \cup F_{3}$ is a cell. If $F_{2}$ and $F_{3}$ do not meet on a facet of $F_{2}$ and $F_{3}$ then $F_{2} \cup F_{3} \cup F_{4}$ would not be a cell, thus $F_{2} \cup F_{3}$ is a cell. Thus $\mathscr{F}_{1} \cap \mathscr{F}_{3}$ splits a facet of $\mathscr{S}^{\sharp}$.

Now by a similar argument $\mathscr{F}_{2} \cap \mathscr{F}_{3}$ splits a facet of $\mathscr{S}^{\sharp}+$ $\mathscr{F}_{1} \cap \mathscr{F}_{3}$ and the result of that splitting is $\mathscr{S}$, thus $\mathscr{S}=\left(\mathscr{S}^{\sharp}+\right.$ $\left.\mathscr{F}_{1} \cap \mathscr{F}_{3}\right)+\mathscr{F}_{2} \cap \mathscr{F}_{3}$.

THEOREM 4. If $\mathscr{S}$ is a nonsimple $d$-gec with at most $d+3$ facets and is not a k-fold pyramid over a simple 2-neighborly gcc, then $\mathscr{S}=\mathscr{S}^{\prime}+\mathscr{C}$ for some subfacet $\mathscr{C}$ of $\mathscr{S}$ and some d-gcc $\mathscr{S}^{\prime}$.

Proof. Again we do only the case where $\mathscr{S}$ has $d+3$ facets. Let $v$ be a nonsimple vertex of $\mathscr{S}$. We consider two cases.

Case I. $d+2$ facets of $\mathscr{S}$ meet $v$. In this case $\mathscr{S}$ is a pyramid over a $(d-1)$-gcc $\mathscr{F}$ which by induction can be written $\mathscr{F}^{\prime}+\mathscr{C}^{\prime}$ for some $(d-1)$-gec $\mathscr{F}^{\prime}$ and some subfacet $\mathscr{C}^{\prime}$ of $\mathscr{F}$. If we let $\mathscr{S}^{\prime}$ be the pyramid over $\mathscr{F}^{\prime}$ and let $\mathscr{C}$ be the pyramid over $\mathscr{C}^{\prime}$ we are done.

Case II. $d+1$ facets of $\mathscr{S}$ meet $v$. In this case ast $(v, \mathscr{S})$ contains two facets meeting on a subfacet. Arguments similar to those in Theorem 3 show that this subfacet is removable.

4. A gec that cannot be generated. The natural question to ask is "can one construct all $d$-gcc's from the $d$-simplex by facet splitting?" That is, given a $d$-gcc $\mathscr{S}$, does there exist a sequence $\mathscr{S}_{1}, \mathscr{S}_{2}, \cdots, \mathscr{S}_{n}$ such that $\mathscr{S}_{n}^{\prime}$ is isomorphic to $\mathscr{S}_{,} \mathscr{S}_{1}$ is isomorphic to the $d$-simplex and each gec is obtained from its predecessor by facet splitting? Although the answer is yes for $d \leqq 3$, it is easy to see that the answer is no for $d \geqq 4$. Consider the cyclic 4-polytope with 6 vertices (see [9]). This is a simplicial polytope with each pair of vertices joined by an edge. This implies that each subfacet lies on two tetrahedra such that an edge joins the two vertices that are not on the subfacet. The subfacet is not removable because if it were, the result of removing the subfacet would be a 4 -gcc with a facet that intersects an edge in two points. By taking pyramids over this polytope we can produce $d$-gcc's with $d+5$ facets that cannot be produced by facet splitting. 
It seems that we should have better luck with simple gec's, however we shall show that there is a simple 4-gec with 10 facets that cannot be generated by facet splitting. We shall use the following lemma.

LEMMA 12. If $\mathscr{S}$ is a simple 2-neighborly 4-gec with $n$ facets, that can be generated by facet splitting, then $\mathscr{S}$ has a subfacet with exactly $n-2$ edges.

Proof. If $\mathscr{S}=\mathscr{S}^{\prime}+\mathscr{C}$ then $\mathscr{S}^{\prime}$ must be 2-neighborly. Suppose $\mathscr{F}$ is the facet of $\mathscr{S}^{\prime}$ that $\mathscr{C}$ splits. Since $\mathscr{S}^{\prime}$ is neighborly $\mathscr{F}$ has $n-2$ facets. If there is a 2 -face $S$ of $\mathscr{F}$ that $\mathscr{C}$ does not split then the facet of $\mathscr{S}$ meeting $\mathscr{F}$ on $S$ will not meet both $\mathscr{F}^{*}$ and $\mathscr{F}^{* *}$. Thus $\mathscr{C}$ splits every 2 -face of $\mathscr{F}$ which implies that $\mathscr{C}$ has $n-2$ edges.

THEOREM 5. There exists a simple 4-gec with 10 facets that cannot be generated by facet splitting.

Proof. We begin with the triangulation of the 3-sphere, $N_{42}^{9}$, of Aultshuler and Steinberg (see [1]). We give a description of $N_{42}^{9}$ in Table 1. The description consists of a list of facets given by their vertices, a list of facets meeting each vertex and finally a matrix whose $i j$ th entry is the valence of edge $i j$, i.e., the number of 2faces containing edge $i j$.

Table 1

The sphere $N_{42}^{9}$

\begin{tabular}{ccc|l|lllllllll}
\hline \multicolumn{3}{c|}{ 3-simplices } & \multicolumn{1}{|c|}{$\begin{array}{c}\text { Simplices with a } \\
\text { given vertex }\end{array}$} & \multicolumn{5}{|c}{ Edge-valence matrix } \\
\cline { 2 - 8 } A-1236 & $\mathrm{J}-1579$ & $\mathrm{~S}-2689$ & 1-ABCDEFGHIJKL & $*$ & 5 & 6 & 4 & 6 & 3 & 5 & 4 & 3 \\
$\mathrm{~B}-1237$ & $\mathrm{~K}-1589$ & $\mathrm{~T}-3458$ & 2-ABCDEMNOPQRS & 5 & $*$ & 3 & 4 & 6 & 7 & 4 & 4 & 3 \\
$\mathrm{C}-1245$ & $\mathrm{~L}-1789$ & $\mathrm{U}-3469$ & 3-ABFGHIMTUVWX & 6 & 3 & $*$ & 6 & 3 & 5 & 6 & 4 & 3 \\
$\mathrm{D}-1246$ & $\mathrm{M}-2367$ & $\mathrm{~V}-3478$ & 4-CDFGNOTUVWY & 4 & 4 & 6 & $*$ & 4 & 5 & 3 & 6 & 4 \\
$\mathrm{E}-1257$ & $\mathrm{~N}-2458$ & $\mathrm{~W}-3479$ & 5-CEFHJKNPQRTS & 6 & 6 & 3 & 4 & $*$ & 3 & 4 & 5 & 5 \\
$\mathrm{~F}-1345$ & $\mathrm{O}-2468$ & $\mathrm{X}-3679$ & 6-ADGMOPQSUXYZ & 3 & 7 & 5 & 5 & 3 & $*$ & 4 & 3 & 6 \\
$\mathrm{G}-1346$ & $\mathrm{P}-2567$ & $\mathrm{Y}-4689$ & 7-BEIJLMPVWXZS & 5 & 4 & 6 & 3 & 4 & 4 & $*$ & 4 & 6 \\
$\mathrm{H}-1358$ & $\mathrm{Q}-2569$ & $\mathrm{Z}-4789$ & 8-HIKLNORSTVYZ & 4 & 4 & 4 & 6 & 5 & 3 & 4 & $*$ & 6 \\
$\mathrm{I}-1378$ & $\mathrm{R}-2589$ & $\mathrm{~S}-5679$ & 9-JKLQRSUWXYZS & 3 & 3 & 3 & 4 & 5 & 6 & 6 & 6 & $*$ \\
\hline
\end{tabular}

This sphere has just one edge, 26, that belongs to seven 2 -faces. We now consider a 3 -cell $C$ in $N_{42}^{9}$, where $C=9826 \cup 9526 \cup 5726 \cup$ $3726 \cup 1372 \cup 9485$. We replace $C$ in $N_{42}^{9}$ by a cell that is the union of simplices $x \vee A$ for each triangle $A$ on the relative boundary of 
$C$, and a point $x$ not in $N_{42}^{9}$. Let $T$ be this new triangulation of the 3-sphere. Since no vertex of $\operatorname{rel} \beta(C)$ has degree greater than 7 , no edge of $T$ will have valence greater than 7 . Of the edges of $N_{42}^{9}$, only 26 could have valence 8 , however, the 2 -cells 926,526 , and 726 were destroyed, thus even edge 26 fails to have valence 8 . Since no edge of $N_{42}^{9}$ lies inside $C$ and since all vertices of $N_{42}^{9}$ lie on $C, T$ is a 2-neighborly triangulation.

The dual of $T$ is our simple 4-gce that cannot be generated by facet splitting (by Lemma 12).

5. Remarks. The methods used in [8] will prove that the simple 4-gcc's up to 8 facets can be generated by facet splitting. Using this, Theorem 4 can be strengthened to include all $d$-gcc's with up to $d+3$ facets except those that are $k$-fold pyramids over a 2-neighborly $(d-k)$-gec for $k \leqq d-5$.

P. Mani [11] has proved that any triangulation of the $d$-sphere with up to $d+4$ vertices is isomorphic to the boundary of a $(d+1)$ polytope. In view of this it is probably true that any simple $d$-gec with up to $d+3$ facets is isomorphic to a $d$-polytope. The author conjectures that this is true for all $d$-gec's with up to $d+3$ facets.

Many theorems that are true for polytopes are also true for gcc's. The lower bound theorem (see [3] and [4]) is true for simple gec's and essentially the same proof as for polytopes proves it. The upper bound conjecture (see [12]) is still open for gec's. Many of the known necessary conditions for a graph to be the graph of a $d$-polytope hold for $d$-gec's (see [4]). Some of these graph theorems were proved independently by $D$. Walkup but never published [private communication].

Amos Altshuler has pointed out that our triangulation $T$ is not 4-polyhedral. Link $(1, T)$ is not stacked, that is, it is not isomorphic to the boundary of a 3-polytope obtained from the simplex by repeatedly adding pyramidal caps. It follows from Theorem 1 of [2] that $T$ is not polyhedral.

The reason for choosing the name generalized combinatorial cell is the following. The author would prefer the term combinatorial cell; it would then be logical to call the boundary of such a cell a combinatorial sphere. However, Grünbaum has used the term combinatorial sphere for triangulations of the sphere [9]. Since our structures are more general than Grünbaum's, we adopted our present terminology.

\section{REFERENCES}

1. A. Altshuler and L. Steinberg, Neighborly combinatorial 3-manifolds with 9 vertices, Discrete Math., 8 (1974), 113-137. 
2. A. Altshuler and L. Steinberg, Neighborly 4-polytopes with 9 vertices, J. Combinatorial Theory, 15 (1973), 270-287.

3. D. Barnette, The minimum number of vertices of a simple polytope, Israel J. Math., 10 (1971), 121-125.

4. - A proof of the lower bound conjecture for convex polytopes, Pacific J. Math., 46 (1973), 349-354.

5. — Graph theorems for manifolds, Israel J. Math., 96 (1973), 62-72.

6. D. Barnette and G. Wegner, A 3-sphere that is not 4-polyhedral, Studia Sc. Math-Hungarica, 6 (1971), 341-346.

7. D. Barnette, Diagrams and Schlegel Diagrams, Combinatorial Structures and Their Applications, Gordon and Breach, New York, 1970.

8. The triangulations of the 3-sphere with up to 8 vertices, J. Combinatorial Theory, 14 (1973), 37-52.

9. B. Grünbaum, On the enumeration of convex polytopes and combinatorial spheres, to appear.

10. Convex Polytopes, Wiley and Sons, New York, 1970.

11. P. Mani, Spheres with few vertices, J. Combinatorial Theory, to appear.

12. P. McMullen, The maximum number of faces of a convex polytope, Mathematica, 17 (1970), 179-184.

13. E. Steinitz and H. Rademacher, Vorlesungen über die Theorie der Polyeder, Springer, Berlin, 1934.

Received April 30, 1974. Research supported by a Sloan Foundation Fellowship and by NSF grant \#GP-27963.

University of California, Davis 
